\title{
Corrigendum: Overlapping Structures Detection in Protein-Protein Interaction Networks Using Community Detection Algorithm Based on Neighbor Clustering Coefficient
}

\author{
Yan Wang ${ }^{1,2}$, Qiong Chen ${ }^{1}$, Lili Yang ${ }^{1,3 *}$, Sen Yang ${ }^{1}, K^{2 i} \mathrm{He}^{1}$ and Xuping Xie ${ }^{1}$ \\ ${ }^{1}$ Key Laboratory of Symbol Computation and Knowledge Engineering of Ministry of Education, College of Computer Science and \\ Technology, Jilin University, Changchun, China, ${ }^{2}$ School of Artificial Intelligence, Jilin University, Changchun, China, ${ }^{3}$ Department \\ of Obstetrics, The First Hospital of Jilin University, Changchun, China
}

Keywords: protein-protein interaction network, overlapping structure, community detection, central edge, clustering coefficient

\section{A corrigendum on}

\section{OPEN ACCESS}

Approved by:

Frontiers Editorial Office, Frontiers Media SA, Switzerland

*Correspondence:

Lili Yang

yllju@jlu.edu.cn

Specialty section:

This article was submitted to

Computational Genomics,

a section of the journal

Frontiers in Genetics

Received: 22 October 2021 Accepted: 01 November 2021 Published: 26 November 2021

Citation:

Wang Y, Chen Q, Yang L, Yang S, He K and Xie $X$ (2021) Corrigendum: Overlapping Structures Detection in Protein-Protein Interaction Networks Using Community Detection Algorithm Based on Neighbor

Clustering Coefficient.

Front. Genet. 12:799818. doi: 10.3389/fgene.2021.799818
Overlapping Structures Detection in Protein-Protein Interaction Networks Using Community Detection Algorithm Based on Neighbor Clustering Coefficient

by Wang, Y., Qiong, C., Yang, L., Yang, S., He, K., and Xie, X. (2021). Front. Genet. 12:689515. doi: 10. 3389/fgene.2021.689515

In the original article, an author name was incorrectly written as "Chen Qiong”. It has been corrected to "Qiong Chen".

Also, there was a mistake in "Funding" section as published. Funding grant numbers for "Development Project of Jilin Province of China" have been changed to "Nos. 20200401083GX, 2020C003,20200403172SF and another funder has been added: "Chinese Postdoctoral Science Foundation (No.801212011421)." The corrected statement appears below: This work was supported by the National Natural Science Foundation of China (Nos. 62072212 and 61902144), the Development Project of Jilin Province of China (Nos. 20200401083GX, 2020C003,20200403172SF) and Chinese Postdoctoral Science Foundation (No.801212011421).

The authors apologize for this error and state that this does not change the scientific conclusions of the article in any way. The original article has been updated.

Publisher's Note: All claims expressed in this article are solely those of the authors and do not necessarily represent those of their affiliated organizations, or those of the publisher, the editors and the reviewers. Any product that may be evaluated in this article, or claim that may be made by its manufacturer, is not guaranteed or endorsed by the publisher.

Copyright $\odot 2021$ Wang, Chen, Yang, Yang, He and Xie. This is an open-access article distributed under the terms of the Creative Commons Attribution License (CC BY). The use, distribution or reproduction in other forums is permitted, provided the original author(s) and the copyright owner(s) are credited and that the original publication in this journal is cited, in accordance with accepted academic practice. No use, distribution or reproduction is permitted which does not comply with these terms. 